\title{
Dor crónica multifatorial e a abordagem holística do médico de família: a propósito de um caso clínico
}

Ana Rita Magalhães, ${ }^{1}$ Ana Peixoto, ${ }^{2}$ Pedro Miguel Teixeira ${ }^{3}$

\section{RESUMO}

Introdução: A dor crónica afeta cerca de 37\% da população adulta portuguesa. É um desafio a todos os clínicos, pela sua natureza multifatorial e a quase sempre necessidade de envolver equipas interdisciplinares na sua abordagem. Os médicos de família (MF), pela posição privilegiada na capacidade de avaliar o utente como um todo, têm um papel preponderante, uma vez que detêm informação privilegiada sobre o ambiente familiar, comunitário e social. Este caso demonstra não só a importância da avaliação holística, mas também da abordagem interdisciplinar, que se traduziu na melhoria da dor autopercecionada inicialmente como um oito para três, segundo a escala numérica (EN).

Descrição do caso: Sexo feminino, 59 anos, empregada hoteleira, antecedentes de artrite reumatoide, obesidade e gonartrosebilateral. Recorre a consulta programada com a sua nova MF por dor de início há anos, agravamento recente, predomínio lombar e membros inferiores, sem fatores de alívio ou agravamento identificáveis, que classifica basal como cinco, com picos de sete/oito na EN. São identificados, como problemas não conhecidos, hipertensão arterial (HTA) não controlada, perturbação depressiva e de ansiedade generalizada, não adesão terapêutica, doença venosa crónica. O acompanhamento decorreu regularmente ao longo de oito meses, com necessidade de investigar causas de HTA não controlada e consequente descoberta de síndroma de apneia do sono, capacitação e empoderamento da utente, tratamento farmacológico e não farmacológico da depressão major, envolvimento de psicóloga, nutricionista e equipa de fisiatria. O controlo da dor não se obteve inicialmente, apesar de terapêutica instituída segundo a escalada analgésica da Organização Mundial da Saúde. Apenas quando todas as patologias foram sendo abordadas de forma efetivamente interdisciplinar foi possível obter o controlo da dor desta utente.

Comentário: Este caso demonstra a importância da avaliação global da pessoa com dor crónica que veio a revelar várias patologias associadas, componente psicossomático marcado e necessidade de abordagens multi e interdisciplinares. Apenas perante a abordagem de todos os problemas de saúde se conseguiu devolver qualidade de vida e capacitação para lidar com as suas doenças, dolências e dor(es).

Palavras-chave: Dor crónica; Depressão; Medicina centrada na pessoa.

\section{INTRODUÇÃO}

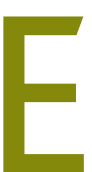

stima-se que a dor crónica afete cerca de $37 \%$ da população adulta portuguesa, sendo, deste modo, a segunda doença crónica mais prevalente, ultrapassada apenas pela HTA. ${ }^{1-2}$ De acordo com o Pain Proposal, ${ }^{3}$ as causas mais comuns de dor crónica na Europa são as patologias da coluna lombar, articulares e cervicais. De acordo com o mesmo estudo, no nosso país a média de tempo em anos decorrente até ao diagnóstico é de 2,5, com $37 \%$ dos doentes com dor crónica a relatar dor não adequadamente controlada. Em Portugal, as principais causas são sobreponíveis, ocupando o terceiro lugar as cefaleias. ${ }^{1}$ Sabe-se que é mais prevalente no sexo feminino, aumentando com a idade e o número de patologias associadas. ${ }^{1}$

1. Médica Assistente de Medicina Geral e Familiar. USF Coimbra Norte, ACeS Baixo Mondego.

2. Médica Assistente de Medicina Geral e Familiar. UCSP Ansião, ACeS Pinhal Interior Norte.

3. Médico Interno de Medicina Geral e Familiar. USF São João Evangelista dos Lóios, ACeS Lisboa Central. 
É um desafio a todos os clínicos, dada a sua natureza multifatorial e a quase sempre necessidade de envolver equipas interdisciplinares na sua abordagem. $\mathrm{O}$ impacto da dor crónica nas atividades de vida diária (AVD) é alarmante, sendo referido por $65 \%$ dos doentes com dor crónica grave, impossibilidade de realizar as mesmas e em $50 \%$ efeitos nas suas relações sociais. ${ }^{3}$ O absentismo laboral é elevado nos portadores de dor crónica, estimando-se, em média, catorze dias por ano nas mulheres e seis dias por ano nos homens. ${ }^{4}$

Os médicos de família (MF) ocupam uma posição privilegiada na capacidade de avaliar o utente como um todo, desconstruindo as partes e unificando o Ser em todo o seu processo. Também na avaliação da dor crónica, o MF tem um papel preponderante, uma vez que, além de se tratar do primeiro ponto de contacto, é também aquele que detém informação privilegiada sobre o ambiente familiar, comunitário e social. ${ }^{5}$

\section{DESCRIÇÃO DO CASO}

Utente do sexo feminino, 59 anos, caucasiana, ateia, natural e residente no concelho de Coimbra. Divorciada há cerca de 12 anos, inserida em família unitária, classe socioeconómica de Graffar média-baixa, trabalha como empregada hoteleira. Apresenta, como antecedentes pessoais conhecidos, artrite reumatoide seguida em consulta de reumatologia no Centro Hospitalar e Universitário de Coimbra (CHUC) e medicada com ledertrexato, obesidade grau 2, gonartroses bilaterais e osteoartroses da coluna lombo sagrada. Plano Nacional de Vacinação (PNV) atualizado, sem vacinas extra PNV. Sem antecedentes familiares relevantes. Tem dois filhos, ambos residentes no concelho de Coimbra, com que contacta frequentemente.

Trata-se de uma utente não frequentadora dos cuidados de saúde primários desde 2013, por relação conflituosa com a MF anterior. Recorre, em outubro de 2018, a consulta programada com a sua nova MF por agravamento das queixas álgicas, de início insidioso há vários anos, que descreve como generalizadas - "dói-me tudo" (sic) -, mas com predomínio nos punhos, coluna lombar e nos membros inferiores (MI), apresentando como fatores de alívio o repouso e como fatores de agravamento identificáveis o esforço físico, o ortostatismo prolongado, o cansaço psicológico (sic) e o final da semana. Refere tratar-se de uma dor tipo moinha, que classifica em nível basal diário como um seis na escala numérica (EN) de dor, com vários picos diários tipo pontada, classificados como sete ou oito na EN. Sem redflags identificados, nomeadamente dor noturna, sintomas sistémicos ou antecedentes de neoplasia. Utiliza, em média, quatro vezes por semana etoricoxib $90 \mathrm{mg}$ como terapêutica de alívio ou diclofenac 75mg. Quando questionada, menciona nem sempre adesão terapêutica ao ledertrexato por efeitos secundários gastrointestinais e por achar que "é muito forte" (sic). Recusou, na última consulta, a administração de biológicos por "não ter tempo para andar a ir para o hospital" (sic).

Verbaliza ainda problemas laborais por mau ambiente com colegas e entidade patronal, mencionando inflexibilidade desta última na sugestão de trabalho adaptado às suas limitações. Mantém, apesar dos 59 anos, uma média de dois turnos noturnos por semana, com elevado desgaste e insónia inicial e de manutenção associada. Associa ainda grande ansiedade antecipatória antes de se dirigir para o local de trabalho com sensação de palpitações, anedonia e desesperança. Por outro lado, apesar dos filhos já não residirem com a mesma, sente-se responsável por eles, tenta ajudá-los financeiramente, cozinha diariamente para toda a família.

Trata-se de uma utente em que é possível perceber a ausência de cuidados alimentares, com obesidade de longa evolução (início após o nascimento do primeiro filho) e de agravamento progressivo, excesso de ingestão de hidratos de carbono e lípidos, apenas fazendo três refeições por dia, sedentária, não pratica qualquer tipo de atividade física programada.

Ao exame objetivo apresentava fácies cansado e olheirento, humor deprimido, anedonia, choro fácil, labilidade emocional, suspiros frequentes. A nível osteoarticular apresentava crepitações finas na entrelinha articular de ambos os punhos, com dor local na mobilização ativa e passiva e ligeira atrofia da eminência tenar esquerda; limitação dos movimentos ativos e passivos da coluna lombar por dor, manobras desacroileíte negativas. Apresentava ainda desvio em varo de ambos os joelhos, crepitações grosseiras na entrelinha articular de ambos os joelhos, sem sinais inflamatórios locais ou edema. Veias varicosas em ambos os MI com ligeiro eczema de estase e edema maleolar bilateral, com pulsos pediosos amplos e simétricos. 
Nesta consulta são, assim, identificados os seguintes problemas não conhecidos: HTA não controlada grau 3 (média das três medições de 190/100mmHg) assintomática; depressão major provavelmente reativa a problemas laborais; insónia; má adesão terapêutica; doença venosa crónica C3/C4 de acordo com a escala CEAP.

O plano conjunto, além da escuta ativa, baseou-se no reforço da importância de uma boa adesão terapêutica, envolvendo as várias áreas e problemas identificados. Por outro lado, foi importante explicar os riscos da utilização sistemática de anti-inflamatórios não esteroides. Nesta consulta, o principal desafio à MF foi evitar a tentação de tentar atuar em todos os problemas identificados, pelo que foi necessário priorizar os mesmos, agendando nova consulta para breve de modo a poder acompanhar adequadamente esta utente. Assim, após ensino, foi acordado para a próxima consulta trazer os resultados da medição ambulatória da pressão arterial (AMPA), tendo-se ainda insistido nas medidas de redução do consumo de sal com entrega de folhetos educativos. Foram prescritas, como medidas não farmacológicas de controlo da dor e peso, caminhadas pelo menos três vezes por semana com calçado adequado e banhos de imersão no final do dia de trabalho. Com o acordo da utente iniciou-se tratamento farmacológico anti-hipertensivo com terapêutica dupla de perindopril $10 \mathrm{mg}+$ amlodipina $5 \mathrm{mg}$ ao jantar e analgesia com paracetamol $325 \mathrm{mg}+$ tramadol $37,5 \mathrm{mg}$, inicialmente id durante cinco dias e depois 2id. Foi ainda prescrito laxante em caso de obstipação e como terapêutica de SOS metamizol $575 \mathrm{mg}$ ou paracetamol $500 \mathrm{mg}$ até 2id. Foram requisitadas análises laboratoriais com hemograma, glicémia, provas de função hepática, renal e tiroideia, perfil lipídico e microalbuminúria em amostra de urina. Adicionalmente foi requisitado doppler venoso dos MI bilateral e eletrocardiograma (ECG). Finalmente, agendou-se consulta para dentro de 15 dias.

Na consulta programada de novembro 2018: dor não controlada (EN média de 5), não cumpre todos os dias a terapêutica, esquece-se frequentemente de uma das tomas de paracetamol+tramadol, usa metamizol como SOS e etoricoxib quando dor exacerbada. Reconhece, contudo, que a medicação melhora a dor quando não se esquece. Nega ainda efeitos secundários da mesma. Não se sente capaz nem motivada a iniciar as cami- nhadas, reforçando que a dor é um grande impedimento, sendo os banhos de imersão fator de alívio da dor. Quando questionada acerca do que espera do MF, verbaliza ser o controlo da dor; porém, quando a questão se foca na própria, ou seja, no que pode fazer para melhorar, sente que não sabe. Menciona que o divórcio foi um período crítico, emocional e financeiramente. Por outro lado, a atual companheira do ex-marido é sua colega de trabalho, em posição de chefia e sente-se prejudicada. Boa relação com os filhos, classifica a relação como muito boa, sendo a estes e a uma amiga a quem recorre quando necessita; todavia, desde que os filhos saíram de casa, há sete e três anos, sente-se mais sozinha. Mantém insónia inicial.

Traz AMPA como solicitado; média de 160/ /100mmHg, garantindo boa adesão, sem esquecimentos. Investigando sinais e sintomas de causas secundárias de HTA, refere roncopatia objetivada pelos filhos (não consegue especificar apneias) e sonolência diurna associada a cefaleias matinais. Aplicado o questionário de Epworth obtém-se uma pontuação de 11 pontos (sonolência anormal).

Dos exames solicitados a salientar LDL de 136mg/dl, ECG com critérios de hipertrofia da aurícula e ventrículo esquerdos e incompetência valvular troncular esquerda no doppler venoso.

Perante cálculo do risco cardiovascular (elevado) e dadas as alterações eletrocardiográficas, são requisitadas provas de esforço, ecocardiograma e doppler carotídeo. Realizado pedido de consulta de pneumologia para estudo do sono e pedido de consulta de cirurgia vascular.

Após plano conjunto, aceita ser encaminhada e acompanhada pelas nutricionista e psicóloga do ACeS. São reforçadas as medidas não farmacológicas de controlo tensional e de alívio da dor e redução de peso, compromete-se a iniciar caminhada e hidroginástica. É providenciado ensino acerca da doença venosa crónica e medidas de alívio e preventivas de agravamento. São prescritas meias elásticas grau 2 de compressão. É aumentada a dose de perindopril+amlodipina para $10 \mathrm{mg}+10 \mathrm{mg}$, iniciada terapêutica com atorvastatina 40mg id e reforçada a importância da adesão terapêutica à medicação analgésica, associando-se duloxetina $60 \mathrm{mg}$ id. Agendou-se consulta para dentro de dois meses. 
O acompanhamento a esta utente decorreu de forma regular ao longo dos seis meses seguintes. No estudo do sono registou-se a síndroma de apneia do sono (SAOS) grave, com uma média de 42 apneias/hipopneias/hora, tendo iniciado uso de CPAP com ótima adesão. Melhoria franca do perfil tensional, apresentando médias de AMPA de 130/70mmHg, mantendo concomitantemente terapêutica anti-HTA. Tem sanefectomia esquerda agendada na cirurgia vascular. A utente iniciou hidroginástica duas vezes por semana $\mathrm{e}$ programa de mindfulness semanal, com controlo da dor sem necessidade de aumento das doses de paracetamol+tramadol 325mg/37,5mg 2id e duloxetina 60mg id, atualmente sem necessidade de terapêutica de SOS e com dor média, segundo a EN, de três pontos. Iniciou também programa de medicina física e de reabilitação com recurso a dois ciclos de 15 sessões de fisioterapia, dirigidos às queixas álgicas osteoarticulares.

A capacitação e empoderamento da utente quanto à necessidade da adesão terapêutica, bem como o envolvimento de psicóloga clínica, nutricionista e equipa de fisiatria/fisioterapia foram determinantes no sucesso da terapêutica farmacológica e não farmacológica. O controlo da dor não se obteve inicialmente, apesar da terapêutica farmacológica instituída, pois apenas quando todas as patologias foram sendo abordadas de forma efetivamente interdisciplinar foi possível obter o controlo da dor desta utente, associando-se também de forma importante o restabelecimento da aliança terapêutica entre MF e utente como pilar basal do sucesso. Durante este processo foram também realizados os rastreios oncológicos do cancro do colo do útero através de citologia e do cancro do cólon por pesquisa de sangue nas fezes, que se encontravam desatualizados e, em ambos, com resultado sem alterações.

\section{COMENTÁRIO}

Este caso permite demonstrar não só a importância da avaliação holística da utente com dor crónica, neste caso uma utente com multimorbilidades, patologia depressiva e dor crónica associada, mas também da abordagem interdisciplinar entre MF, equipa de fisiatria, nutricionista e psicólogo que se traduziu numa melhoria, em cerca de oito meses, de uma dor autopercecionada como um oito, segundo a EN, para uma dor três. Todos os restantes componentes foram ava- liados e orientados pelo MF, o que permitiu além do controlo da dor crónica, o controlo da HTA, a orientação da SAOS e da doença venosa crónica, da patologia depressiva, das estratégias de coping e, em última análise, da qualidade de vida da utente, muito comprometida até então. Apenas perante a resolução/abordagem de todos os problemas de saúde como um todo se conseguiu devolver qualidade de vida e capacitação para lidar com as suas doenças, dolências e dor(es).

É, assim, demonstrada a importância da avaliação global de uma utente com dor crónica que veio a revelar várias patologias associadas, componente psicossomático marcado e necessidade de abordagens multi e interdisciplinares.

Como pontos fortes, a autora considera a importância da perspetiva global que este caso clínico aborda, nomeadamente a interação entre todos os profissionais envolvidos e a capacidade unificadora que o MF representa. Por outro lado, também o facto de se ter tentado sempre praticar uma medicina centrada na pessoa, e não na doença, permitiu o sucesso terapêutico com uma abordagem biopsicossocial. Como pontos menos positivos, a principal dificuldade sentida foi, sem dúvida, a dispersão de conteúdos e as inúmeras, mas inevitáveis, frentes de atuação em que a MF se viu obrigada a intervir. Por outro lado, instrumentos padronizados de avaliação familiar e social, como o Círculo de Thrower ou o Ecomapa, poderiam enriquecer a avaliação holística da utente; porém, o escasso tempo de consulta foi sendo canalizado para a gestão de todas as morbilidades.

\section{REFERÊNCIAS BIBLIOGRÁFICAS}

1. Azevedo LF, Costa-Pereira A, Mendonça L, Dias CC, Castro-Lopes JM. Epidemiology of chronic pain: a population-based nationwide study on its prevalence, characteristics and associated disability in Portugal. J Pain. 2012;13(8):773-83.

2. Polonia J, Martins L, Pinto F, Nazare J. Prevalence, awareness, treatment and control of hypertension and salt intake in Portugal: changes over a decade. The PHYSA study. J Hypertens. 2014;32(6):1211-21.

3. Baker M, Collett B, Fischer A, Hermann V, Huygen F, Tölle T, et al. Pain proposal: improving the current and future management of chronic pain (a European consensus report). Brussels: Pfizer; 2010.

4. Azevedo LF. Dor crónica em Portugal: prevalência e impacto individual, social e económico [dissertation]. Porto: Faculdade de Medicina da Universidade do Porto; 2013.

5. WONCA Europa. A definição europeia de medicina geral e familiar (Clínica geral / Medicina familiar). WONCA Europa; 2002. Available from: 
6. Allen J, Gay B, Crebolder H, Heyrman J, Svab I, Ram P. The European definition of general practice/family medicine [Internet]. In: SemFYC, EURACT. Barcelona:WONCA; 2011.Available from: https://www.woncaeurope.org/ file/520e8ed3-30b4-4a74-bc35-87286d3de5c7/Definition\% 203rd\%20ed\%202011\%20with\%20revised\%20wonca\%20tree.pdf

\section{CONFLITO DE INTERESSES}

Os autores declaram não ter quaisquer conflitos de interesse.

\author{
ENDEREÇO PARA CORRESPONDÊNCIA \\ Ana Rita Magalhães \\ E-mail: anaritamag@gmail.com \\ https://orcid.org/0000-0001-5579-9276
}

Recebido em 12-08-2019

Aceite para publicação em 08-04-2020

\section{ABSTRACT}

\section{MULTIFACTORIAL CHRONIC PAIN AND THE HOLISTIC APPROACH OF THE GENERAL PRACTITIONER: ABOUT A CLINICAL CASE}

Introduction: Chronic pain affects about $37 \%$ of the Portuguese adult population. It's a challenge for all clinicians because of its multifactorial nature and the almost always need to involve interdisciplinary teams in its approach. General practitioners (GP) because of their privileged position in the ability to evaluate the patient as a whole, play a leading role, since they also have privileged information about the family, community, and social environment. This case demonstrates not only the importance of the holistic assessment but also the interdisciplinary approach that translated into an improvement, from self-perceived pain as an eight, according to the numerical scale, to a three.

Case description: Female, 59 years old, hotel employee, history of rheumatoid arthritis, obesity, and bilateral gonarthrosis. She scheduled a consultation with her new GP because of lumbar and lower limb pain with years of onset, recent worsening, with no identifiable relief or aggravation factors, which she classifies baseline as a five, with peaks of seven/eight in NS. The following are identified as unknown problems: uncontrolled hypertension, depressed and generalized anxiety disorder, nonadherence, chronic venous disease. Follow-up was conducted regularly over eight months, with the need to investigate causes of uncontrolled hypertension and consequent discovery of sleep apnea syndrome, patient empowerment, pharmacological and nonpharmacological treatment of major depression, the involvement of psychologist, nutritionist and physiatrics team. Pain control was not initially achieved despite therapy instituted according to the World Health Organization analgesic escalation. Only when all the pathologies were being effectively addressed in an interdisciplinary way was it possible to obtain pain control of this patient.

Comment: This case demonstrates the importance of the global assessment of the person with chronic pain that has revealed several associated pathologies, marked psychosomatic components, and the need for multi and interdisciplinary approaches. Only by addressing all health problems was it possible to giving her quality of life and enabling her to cope with her illnesses, illnesses, and pain(s).

Keywords: Chronic pain; Depression; Patient-centered medicine. 